Document downloaded from:

http://hdl.handle.net/10251/39858

This paper must be cited as:

Pizá, R.; Salt Llobregat, JJ.; Sala, A.; Cuenca Lacruz, AM. (2014). Hierarchical TripleMaglev Dual-Rate Control Over a Profibus-DP Network. IEEE Transactions on Control Systems Technology. 22(1):1-12. doi:10.1109/TCST.2012.2222883.

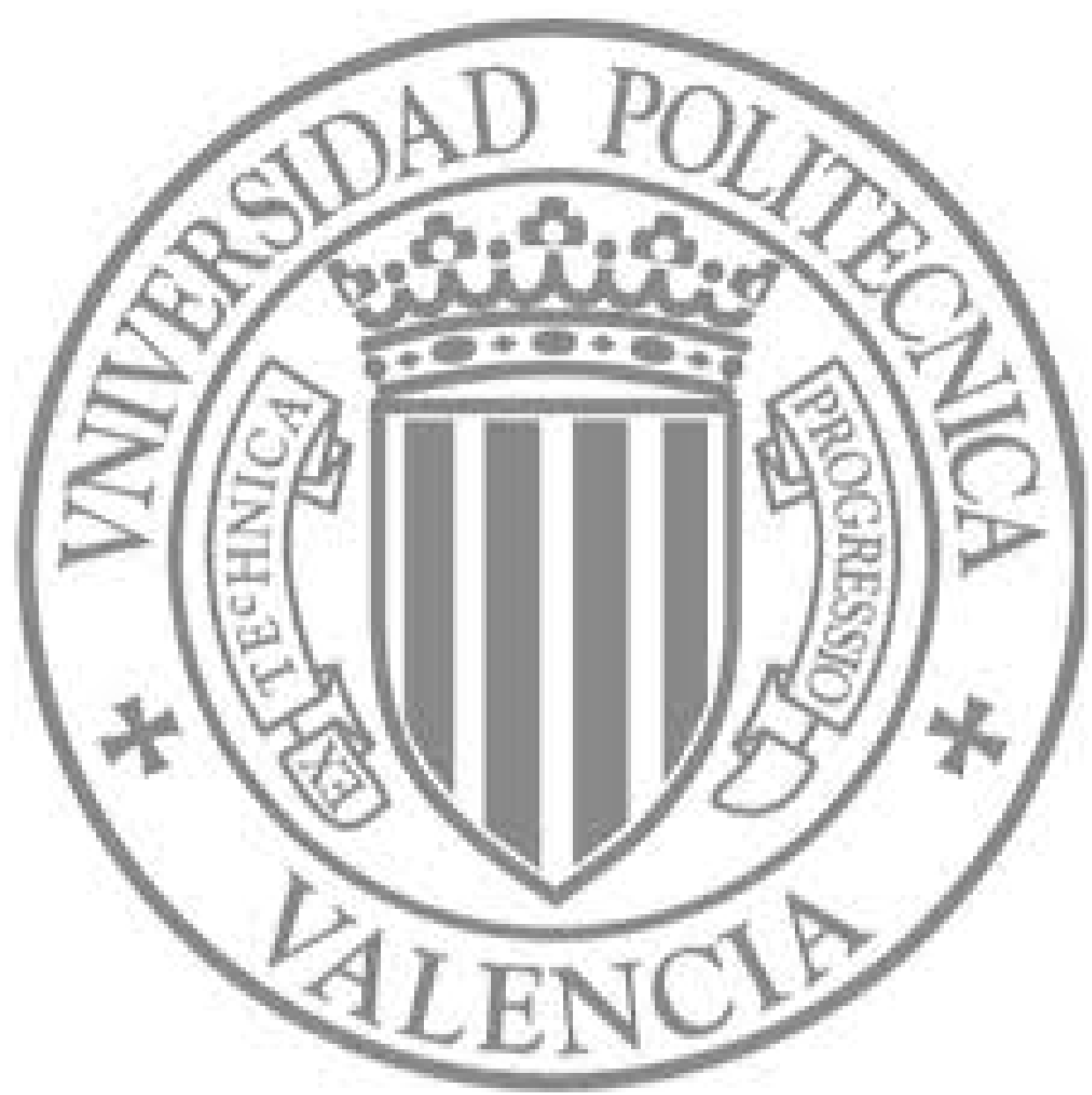

The final publication is available at

http://dx.doi.org/10.1109/TCST.2012.2222883

Copyright Institute of Electrical and Electronics Engineers (IEEE) 


\title{
Hierarchical triple-Maglev Dual-Rate Control over a Profibus-DP Network
}

\author{
Ricardo Pizá, Julián Salt, Member, IEEE, Antonio Sala, Member, IEEE, Ángel Cuenca, Member, IEEE
}

\begin{abstract}
This paper addresses a Networked Control System (NCS) application on an unstable triple-magnetic levitation setup. A hierarchical dual-rate control using a Profibus-DP network has been used in order to stabilise a triangular platform composed of three maglevs. The control difficulty is increased due to the existence of time-varying network-induced delays. To solve this issue, a local decentralised $\mathcal{H}_{\infty}$ control action is complemented by means of a lower-rate output-feedback controller in the remote side. Experimental results show a good stabilization and reference position accuracy under disturbances.
\end{abstract}

Index Terms-Networked control systems, network delay, dualrate control systems, LMI, stability analysis, maglev, Profibus.

\section{INTRODUCTION}

When a control application is projected on a network based environment, in which different devices (sensor, actuator, controller) are connected by means of a shared communication medium [1], [2], [3], typical problems such as data packet losses, lack of synchronization among devices, bandwidth limitations, and time-varying delays occur.

In some cases, the controlled process is very sensitive to these problems.

That is the case of a maglev based platform [4], [5], in network based control [6]. This magnetic levitation process control problem is challenging because each one of the systems that configure the platform are unstable and nonlinear. Maglev platforms have also been used to demonstrate applicability of control strategies in research literature [7], [8].

Also, magnetic levitation has a wide range of applications [9], [10]. Thus, this paper demonstrates some network-based hierarchical control techniques in a 3-maglev platform.

Regarding the network to be selected in control, there are plenty of options with current technology. In an unstable, fast system such as the one in consideration, a low-latency highbandwidth network would be a reasonable choice, for example a network based on traditional field bus protocols such as Profibus, DeviceNet, CAN, InterBus, Field Bus... or a network based on newer industrial Ethernet protocols as ProfiNET, EtherNet/IP, Powerlink, EtherCAT, Modbus TCP or SERCOS

The authors are with Departamento de Ingeniería de Sistemas y Automática, Instituto Universitario de Automática e Informática Industrial, Universitat Politecnica de Valencia, Camino de Vera s/n, 46022 Valencia (Spain). E-mail: \{rpiza, julian, asala, acuenca\}@ isa.upv.es

A. Cuenca, R. Pizá and J. Salt are grateful to the financial support of Spanish Ministerio de Economía research grants DPI2011-28507-C0201, DPI2009-14744-C03-03 and ENE2010-21711-C02-01 and Generalitat Valenciana grant $\mathrm{GV} / 2010 / 018$.

A. Sala is grateful to the financial support of Spanish Ministerio de Economía research grant DPI2011-27845-C02-01, and Generalitat Valenciana grant PROMETEO/2008/088.

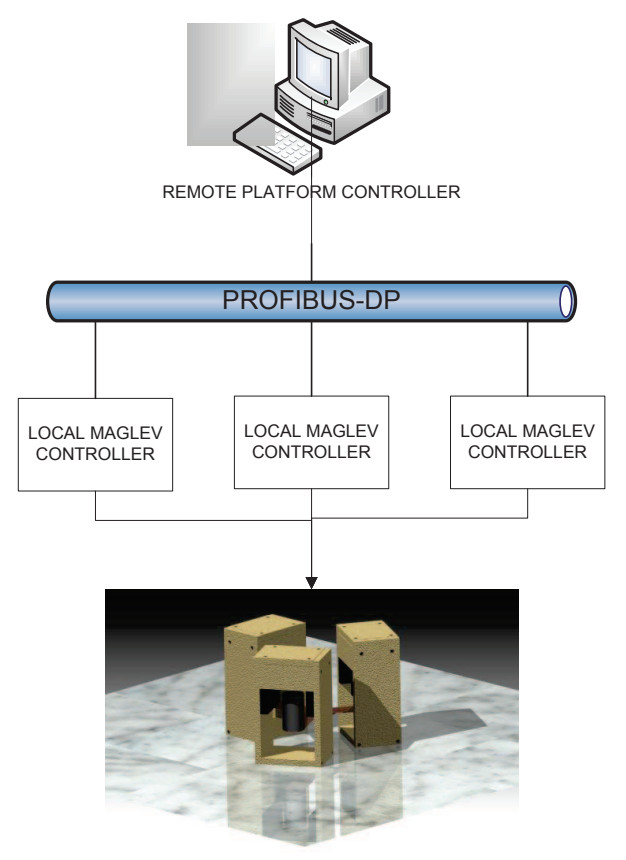

Fig. 1. Hierarchical control structure

III [11]. Any of these networks may be suitable for controlling this platform.

In this work a Profibus-DP with asynchronous operation mode has been selected [12]. Some characteristics of Profibus make this network specially interesting and challenging from a control engineering point of view. There are two kinds of delays on this network: one of them is the delay induced by data transfer between buffers and nodes, and the other one is the delay that appear in the transmission between network nodes; hence, a distribution of time-varying communication delay appears. The chosen control strategy will also require triggered bus mechanism for synchronization based on Profibus freeze and sync commands. Other Profibus-based control applications are reported in [13], [14], etc.

The objective of the paper is to experimentally demonstrate a methodology, described below, for designing hierarchical network based control systems for a multivariable, unstable plant when computational capabilities and network bandwidth/sampling rates are restricted, and data transfer delays are present.

So, a hierarchical control system is proposed. Indeed, due to the limitations in sampling rate and the communication delay magnitudes it was not possible to design a single stabilizing remote control in the maglev platform. Consequently, first 
of all, a fast-rate local decentralised controller is designed and, in the following step, a more sophisticated slower-rate "coordinating" remote control has been designed. Indeed, due to the lack of shared information, the decentralised controllers (perfectly working with one maglev) had a bad performance with the 3-maglev platform in place, falling frequently: neither the local-only nor the remote-only solutions were satisfactory.

For each maglev a standalone local controller has been designed and implemented using robust $\mathcal{H}_{\infty}$ control techniques [15]. The remote controllers have been designed using LMIgridding techniques [16].

The paper is organised as follows. Second section describes the physical process (the triple-maglev system) used as a test platform in the experiments. Third section (and an additional appendix) presents how this process is modeled and linearised. The hierarchical control structure is introduced in section IV. Before presenting the experimental results in section VI, some network and hardware configuration aspects used in these experiments are detailed in section V. Finally, a conclusion section is included.

\section{3-MagleV Platform Description}

The experimental platform used in this work comprises several elements:

- Three magnetic levitation units. These units can be operated in a standalone way, as done in experiment 1 in later sections.

- A Y-shaped levitated platform to which individual maglevs can be attached.

- A National Instruments CompactRio 9074 acting as a local controller.

- A desktop PC acting as a remote controller.

- A Profibus-DP network.

Let us describe now the most relevant characteristics of them.

The controlled plant is a levitated platform, shown in Fig. 2 (left). This levitated platform is an equilateral triangle shape with permanent magnets located at the corners of the platform. A drawing scheme is shown in Fig. 3. The vertical position of each magnet is controlled by an electro-magnet. Thus, there are three maglevs located at the terminals of the platform. Each maglev takes a voltage input signal to generate the magnetic field and takes the vertical position measure using a set of infrared sensor array.

The levitators have been provided by Extra Dimension Technologies (http://www.xdtech.com), model ML-EA. The magnetic levitation unit includes the own levitator with his own power amplifier unit. The maglev provides position information, from the infrared sensor array in \pm 10 volts range. The control signal to be provided to the power amplifier must be also in \pm 10 volts range.

In order to build the coupled platform, the three independent loads of the maglevs were attached to the above referred Y-shape aluminum sheet. Fig. 4 shows a drawing with the levitated structure. Dimensions are expressed in $\mathrm{mm}$, and in the center of the platform the circular shapes correspond to an
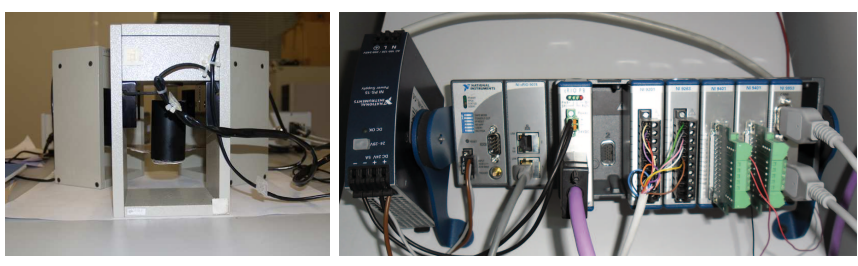

Fig. 2. Experimental setup.

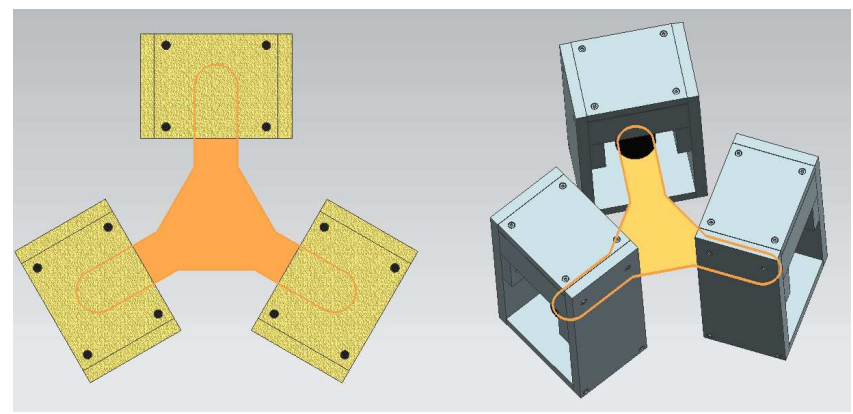

Fig. 3. Experimental setup. Drawings

extra load (a coin of 2 euros, 8.5 grams) used in transient analysis experiments. The total weight for the levitated platform is $0.423 \mathrm{Kg}$.

Further details on the control-relevant model parameters are discussed in appendix A.

A similar maglev platform can be found in different works, for example, in [17], as well as those based on predictive control [18], fuzzy control [19], [20], or some non-linear control methods [21].

\section{MODELLING AND LINEARIZATION}

This section discusses how the specific model is obtained and linearised in order to apply the control scheme proposed in later sections.
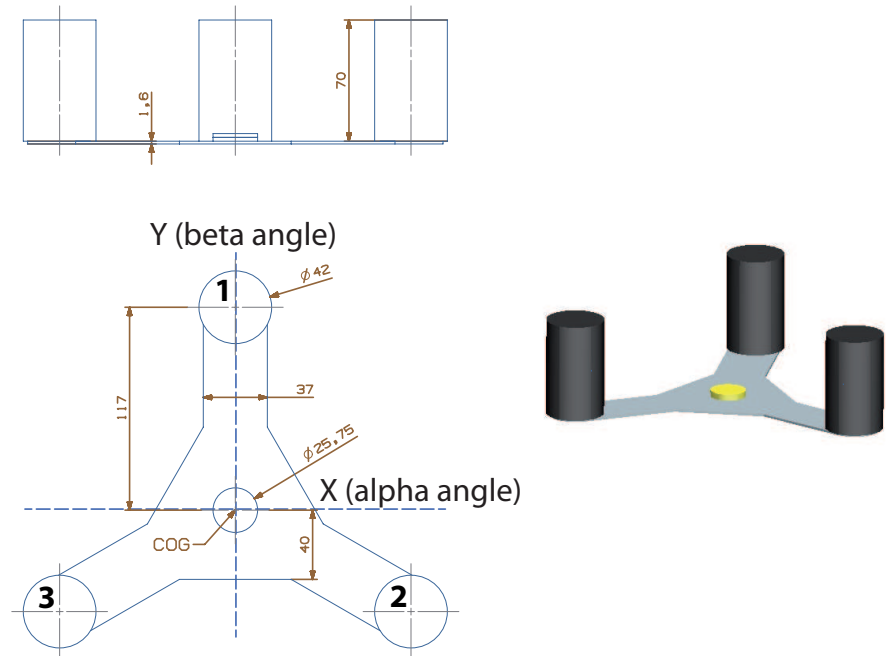

Fig. 4. Platform dimensions (top and side view), reference model axis and maglev numeration. 


\section{A. Single Maglev Modeling}

The well-known Lagrange equations [22] can be used to derive the differential equations of electro-mechanical systems

$$
\frac{d}{d t} \frac{\partial T}{\partial \dot{q}}-\frac{\partial T}{\partial q}+\frac{\partial D}{\partial \dot{q}}+\frac{\partial V}{\partial q}=F_{G}
$$

where $T, V$ and $D$ represent the generalized kinetic ${ }^{1}$, and the potential and dissipation energies, respectively. $F_{G}$ denotes the generalized forces, and the variable $q$ express one of the generalized coordinates.

Let us denote the air gap between the variable magnet and the levitated load with the variable $z$. Assuming infinite permeability except on air gap, with permeability $\mu$, and uniform magnetic flux density $B$, the energy $T_{m a g}$ stored in the levitator's air gap is:

$$
T_{\text {mag }}=\frac{B^{2}}{2 \mu} \cdot A \cdot z
$$

where $A$ is the cross-sectional area, being $B$ given by $F_{m} \mu / z$, where $F_{m}$ stands for the magneto-motive force (linear with current $N I$ (coil) plus a permanent magnet component $I_{0}$, being $N$ the inductor number of turns).

In summary, the magnetic energy is:

$$
T_{\text {mag }}=\frac{\left(N I+I_{0}\right)^{2} \mu A}{2 z}
$$

The reader is referred to [23] (for instance) for details on magnetic field modeling.

Adding the mechanical kinetic energy term $T_{\text {mech }}=\frac{1}{2} m \dot{z}^{2}$, the gravitational potential $V=-m g z$, the resistive dissipation $R \cdot i$, and using both charge and air gap as generalised coordinates, the following nonlinear model can be obtained (details omitted for brevity, see [24] for similar modelling problems):

$$
\begin{array}{r}
m \ddot{z}-\frac{\mu\left(N I+I_{0}\right)^{2} A}{4 z^{2}}-m g=0 \\
\frac{\mu A N^{2}}{z} \dot{I}+\frac{\mu A N\left(I_{0}+N I\right) \dot{z}}{z^{2}}+R I=V
\end{array}
$$

Of course, the uniform-magnetic-field model is not exact as there exist lateral and bottom air gaps. In addition, if the intensity polarity were abruptly changed, the flux lines for the resulting magnetic field would be modified; however, the assumption is made that such situations will not occur around the chosen operation point.

The equations obtained before, (2)-(3), define the theoretical model. Assuming a suitable operating point, a linearized single maglev continuous-time model is obtained:

$$
\begin{array}{r}
\frac{M}{3} \cdot \ddot{z}(t)=f(t) \\
f(t)=K_{1} I(t)+K_{2} \cdot z(t) \\
L \dot{I}(t)+Q \cdot \dot{z}(t)+R I(t)=v(t)
\end{array}
$$

where $M / 3$ is the mass of the whole levitated platform corresponding to one levitator, $f$ is the electromagnetic force,

\footnotetext{
${ }^{1}$ To avoid confusion, note that $T$ will later denote sampling rate, but symbolising in this way kinetic energy keeps notation in this section similar to standard mechanics textbooks.
}

the constants $L, R, K_{1}$ and $K_{2}$ are available in the levitator's manual provided by the manufacturer. The parameter $Q$ models the potential induced by the movement of the levitated magnet ${ }^{2}$. The maglev's numerical values for the parameters can be consulted in Appendix A.

Introducing a measurement equation

$$
y(t)=K_{3} \cdot z(t)
$$

the variable $y$ is the position measurement taken with the infrared sensor system, being $K_{3}$ a linearised calibration constant available on the maglev user's manual.

These equations can be expressed in state space form for a generic maglev $i$ as:

$$
\begin{array}{r}
\left(\begin{array}{c}
\dot{I}_{i}(t) \\
\dot{z}_{i}(t) \\
\ddot{z}_{i}(t)
\end{array}\right)=\left(\begin{array}{ccc}
\frac{-R_{i}}{L_{i}} & 0 & \frac{-Q^{i}}{L_{i}} \\
0 & 0 & 1 \\
\frac{3 K_{1}^{i}}{M} & \frac{3 K_{2}^{i}}{M} & 0
\end{array}\right) \\
\\
+\left(\begin{array}{c}
I_{i}(t) \\
z_{i}(t) \\
\dot{z}_{i}(t)
\end{array}\right)+ \\
y_{i}(t)=\left(\begin{array}{lll}
0 & K_{3}^{i} & 0 \\
0 \\
0
\end{array}\right) \cdot v_{i}(t) \\
\end{array}
$$

\section{B. Global Platform (coupled) Model}

When the global three-levitator platform is considered, its position will be determined by the height of the center of mass and by two angular coordinates ${ }^{3}$. Rigid-body dynamic equations must be then considered.

First of all, the meaning of $z$ will be changed to denote the vertical displacement of the platform's center-of-mass ( $z_{i}$ will denote each maglev's). The linear motion is only considered along the $z$-axis:

$$
M \ddot{z}=\sum_{i} f_{i}
$$

Second, denoting the resulting torque vector as $\tau$, the rotation of the platform around the center of gravity yields

$$
J \dot{\omega}=\tau-\omega \times(J \omega)
$$

where $J$ is the inertia matrix. However, when linearizing around zero rotational speed, and choosing small rotations around principal axis (diagonal inertia matrix) as angular coordinates, then these equalities can be considered, being $\alpha$ and $\beta$ the angles of rotation of levitated platform around $X$ and $Y$ axes respectively.

\footnotetext{
${ }^{2}$ It has been estimated in a separate experiment by moving the maglev and measuring position and induced voltage (opening the circuit). A least-squares fit between a filtered numerical derivative of the position and the voltage has been used (details omitted for brevity).

${ }^{3}$ The rest of the degrees of freedom, i.e., rotation around the vertical axis and two horizontal displacement coordinates, cannot be controlled with the available actuators. Fortunately, as the levitators are somehow attracted towards the electromagnets also in the horizontal axis, these uncontrollable models are stable. Nevertheless they are only very lightly damped (by air, eddy currents...; actually, damping is so subtle that it has not been considered in the -controlled- vertical coordinate movement): the platform must be carefully and slowly positioned without introducing significant energy on these coordinates.
} 


$$
\begin{aligned}
& J_{x x} \ddot{\alpha}=\tau_{x} \\
& J_{y y} \ddot{\beta}=\tau_{y}
\end{aligned}
$$

being $\tau_{x}$ and $\tau_{y}$ the torques on axis $x$ and $y$, respectively, with the chosen reference frame as shown in Fig. 4.

Expressions of torques at the center of gravity from each of the maglev electromagnetic forces are:

$$
\begin{array}{ll}
\tau_{1 x}=f_{1} L \cos \alpha, & \tau_{1 y}=0 \\
\tau_{2 x}=f_{2} L \cos \alpha \cos \beta \sin \frac{\pi}{6}, & \tau_{2 y}=f_{2} L \cos \alpha \cos \beta \sin \frac{\pi}{3} \\
\tau_{3 x}=f_{3} L \cos \alpha \cos \beta \sin \frac{\pi}{6}, & \tau_{3 y}=f_{3} L \cos \alpha \cos \beta \sin \frac{\pi}{3}
\end{array}
$$

being $L$ the length of the arm, and $\alpha$ and $\beta$ the rotation angles around $\mathrm{X}$ and $\mathrm{Y}$ axes respectively.

For sensor measurements, these are the expressions:

$$
\begin{aligned}
& z_{1}=z+L \alpha \\
& z_{2}=z-L \sin \frac{\pi}{6} \cdot \alpha+L \sin \frac{\pi}{3} \cdot \beta \\
& z_{3}=z+L \sin \frac{\pi}{6} \cdot \alpha-L \sin \frac{\pi}{3} \cdot \beta
\end{aligned}
$$

Then, the equation of movement in the vertical direction is:

$$
M \ddot{z}=f_{1}+f_{2}+f_{3}
$$

The linearized torques around the horizontal position are:

$$
\begin{array}{ll}
\tau_{1 x}=f_{1} L, & \tau_{1 y}=0 \\
\tau_{2 x}=f_{2} L \sin \frac{\pi}{6}, & \tau_{2 y}=f_{2} L \sin \frac{\pi}{3} \\
\tau_{3 x}=f_{3} L \sin \frac{\pi}{6}, & \tau_{3 y}=f_{3} L \sin \frac{\pi}{3}
\end{array}
$$

Hence, the overall linearized system equations are:

$L_{1} \dot{I}_{1}+Q_{1} \cdot \dot{z}_{1}+R_{1} I_{1}=v_{1}$

$L_{2} \dot{I}_{2}+Q_{2} \cdot \dot{z}_{2}+R_{2} I_{2}=v_{2}$

$L_{3} \dot{I}_{3}+Q_{3} \cdot \dot{z}_{3}+R_{3} I_{3}=v_{3}$

$f_{1}=K_{1}^{1} I_{1}+K_{2}^{1} z_{1}=K_{1}^{1} I_{1}+K_{2}^{1} L \alpha$

$f_{2}=K_{1}^{2} I_{2}+K_{2}^{2} z_{2}=K_{1}^{2} I_{2}+K_{2}^{2} L\left(\sin \frac{\pi}{3} \cdot \beta-\sin \frac{\pi}{6} \cdot \alpha\right)$

$f_{3}=K_{1}^{3} I_{3}+K_{2}^{3} z_{3}=K_{1}^{3} I_{3}+K_{2}^{3} L\left(\sin \frac{\pi}{6} \cdot \alpha-\sin \frac{\pi}{3} \cdot \beta\right)$

$M \ddot{z}=f_{1}+f_{2}+f_{3}$

$J_{x x} \ddot{\alpha}=L\left(f_{1}+f_{2} \sin \frac{\pi}{6}+f_{3} \sin \frac{\pi}{6}\right)$

$J_{y y} \ddot{\beta}=L\left(f_{2} \sin \frac{\pi}{3}+f_{3} \sin \frac{\pi}{3}\right)$

Reorganizing:

$\dot{I}_{1}=-\frac{R_{1}}{L_{1}} I_{1}-\frac{Q_{1}}{L_{1}} \dot{z}-\frac{Q_{1} L}{L_{1}} \dot{\alpha}+\frac{1}{L_{1}} v_{1}$

$\dot{I}_{2}=-\frac{R_{2}}{L_{2}} I_{2}-\frac{Q_{2}}{L_{2}} \dot{z}+\frac{Q_{2} L \sin \frac{\pi}{6}}{L_{2}} \dot{\alpha}-\frac{Q_{2} L \sin \frac{\pi}{3}}{L_{2}} \dot{\beta}+\frac{1}{L_{2}} v_{2}$

$\dot{I}_{3}=-\frac{R_{3}}{L_{3}} I_{3}-\frac{Q_{3}}{L_{3}} \dot{z}-\frac{Q_{3} L_{\sin } \frac{\pi}{6}}{L_{3}} \dot{\alpha}+\frac{Q_{3} L_{\sin } \frac{\pi}{3}}{L_{3}} \dot{\beta}+\frac{1}{L_{3}} v_{3}$

$\ddot{z}=\frac{K_{1}^{1}}{M} I_{1}+\frac{K_{1}^{2}}{M} I_{2}+\frac{K_{1}^{3}}{M} I_{3}+\frac{L\left(K_{2}^{1}+\sin \frac{\pi}{6}\left(K_{2}^{3}-K_{2}^{2}\right)\right)}{M} \alpha+$

$+\frac{L \sin \frac{\pi}{3}\left(K_{2}^{2}-K_{2}^{3}\right)}{M} \beta$

$\ddot{\alpha}=\frac{L K_{1}^{1}}{J_{x x}} I_{1}-\frac{L K_{1}^{2}}{J_{x x}} I_{2}-\frac{L K_{1}^{3}}{J_{x x}} I_{3}+$

$+\frac{L^{2}\left(K_{2}^{1}+\sin ^{2} \frac{\pi}{6}\left(K_{2}^{2}-K_{2}^{3}\right)\right)}{J_{x x}} \alpha+\frac{L^{2} \sin \frac{\pi}{6} \sin \frac{\pi}{3}\left(K_{2}^{3}-K_{2}^{2}\right)}{J_{x x}} \beta$

$\ddot{\beta}=\frac{L K_{1}^{2} \sin \frac{\pi}{3}}{J_{y y}} I_{2}+\frac{L K_{1}^{3} \sin \frac{\pi}{6}}{J_{y y}} I_{3}+$

$+\frac{L^{2} \sin \frac{y}{6}\left(K_{2}^{3} \sin \frac{\pi}{6}-K_{2}^{2} \sin \frac{\pi}{3}\right)}{J_{y y}} \alpha+$

$+\frac{L^{2} \sin \frac{\pi}{3}\left(K_{2}^{2} \sin \frac{\pi}{3}-K_{2}^{3} \sin \frac{\pi}{6}\right)}{J_{y y}} \beta$

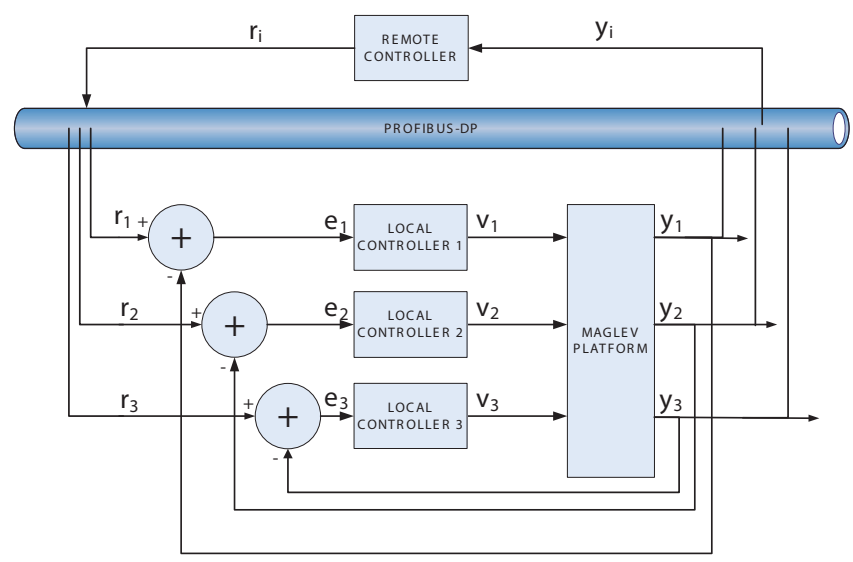

Fig. 5. Proposed hierarchical control system architecture. Remote supervisory level controller, communications network, local controllers and maglev platform.

Therefore, denoting the state, input and output vector as:

$$
x=\left(\begin{array}{c}
I_{1} \\
I_{2} \\
I_{3} \\
z \\
\dot{z} \\
\alpha \\
\dot{\alpha} \\
\beta \\
\dot{\beta}
\end{array}\right), u=\left(\begin{array}{c}
v_{1} \\
v_{2} \\
v_{3}
\end{array}\right), y=\left(\begin{array}{c}
K_{3}^{1} z_{1} \\
K_{3}^{2} z_{2} \\
K_{3}^{3} z_{3}
\end{array}\right)
$$

then the state equations are:

$$
\begin{array}{r}
\dot{x}=A x+B u \\
y=C x
\end{array}
$$

being the system matrices those in (14) on page 5 .

\section{Discrete DuAl-Rate Closed-LOOP MODELS AND CONTROL STRUCTURE}

If the linear state equations (12) and (13) are discretized at a period $T_{0}$ (with zero-order hold at the input), it results in:

$$
\begin{aligned}
x\left((k+1) T_{0}\right) & =A x\left(k T_{0}\right)+B u\left(k T_{0}\right) \\
y\left(k T_{0}\right) & =C x\left(k T_{0}\right)+D u\left(k T_{0}\right)
\end{aligned}
$$

The state of each of the subsystems (to be used by each decentralised controller) will be denoted as $x_{i}$ in the sequel, and the output as $y_{i}$. Obviously, the full state $x$ above is composed by the juxtaposition of all $x_{i}$ and, due to the inertial coupling, matrix $A$ is not block-diagonal.

Consider now the hierarchical control structure appearing in Figure 5. This structure depicts multiple controllers controlling in a decentralised way the above plant, and a remote controller in charge of coordination.

Each subsystem has a one degree of freedom local controller attached to it, whose state will be denoted as $X_{C i}$, with equations given by: 


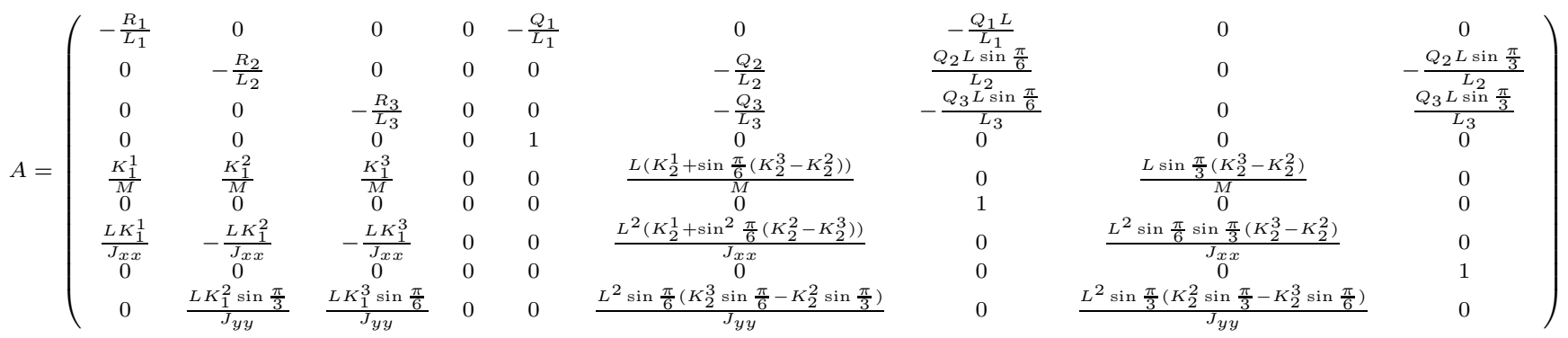

$B=\left(\begin{array}{ccc}\frac{1}{L_{1}} & 0 & 0 \\ 0 & \frac{1}{L_{2}} & 0 \\ 0 & 0 & \frac{1}{L_{3}} \\ 0 & 0 & 0 \\ 0 & 0 & 0 \\ 0 & 0 & 0 \\ 0 & 0 & 0 \\ 0 & 0 & 0 \\ 0 & 0 & 0\end{array}\right) C=\left(\begin{array}{ccccccccc}0 & 0 & 0 & K_{3}^{1} & 0 & K_{3}^{1} L & 0 & 0 & 0 \\ 0 & 0 & 0 & K_{3}^{2} & 0 & -K_{3}^{2} L \sin \frac{\pi}{6} & 0 & K_{3}^{2} L \sin \frac{\pi}{3} & 0 \\ 0 & 0 & 0 & K_{3}^{3} & 0 & K_{3}^{3} L \sin \frac{\pi}{6} & 0 & -K_{3}^{3} L \sin \frac{\pi}{3} & 0\end{array}\right)$

$$
\begin{aligned}
X_{C i}\left((k+1) T_{0}\right) & =A_{d}^{[i]} X_{C i}\left(k T_{0}\right)+B_{d}^{[i]} e_{i}\left(k T_{0}\right) \\
v_{i}\left(K T_{0}\right) & =C_{d}^{[i]} X_{C i}\left(k T_{0}\right)+D_{d}^{[i]} e_{i}\left(k T_{0}\right)
\end{aligned}
$$

being $e_{i}=r_{i}-y_{i}$ or, considering all of them in vector notation, $e=r-y$.

Obviously, the overall controller state equations of the local subsystem will have a block-diagonal structure: the notation $X_{C}$ will denote the state of all controllers (juxtaposing each controller state in a larger vector). The state of the controlled system (3-maglev platform) plus that of the local controllers will be denoted as $\chi=\left(\begin{array}{ll}x^{T} & X_{C}^{T}\end{array}\right)^{T}$. Obviously, although the controller state equations are block-diagonal, there will be some coupling due to the plant not being fully diagonal. The role of the remote controller will be to compensate such coupling neglected in the local decentralized side.

In order to achieve the coordination, the overall local subsystem equations will be considered in the form:

$$
\begin{aligned}
\chi\left((k+1) T_{0}\right) & =A_{0} \chi\left(k T_{0}\right)+B_{0} r\left(k T_{0}\right) \\
y\left(k T_{0}\right) & =C_{0} \chi\left(k T_{0}\right)+D_{0} r\left(k T_{0}\right)
\end{aligned}
$$

\section{A. Dual-rate modelling}

The remote system will be in charge of controlling the local subsystem over a network at a slower rate, because network limitations will be assumed to exist limiting the sampling rate (see section $\mathrm{V}$ ).

The local controllers will be operating at a fast sampling rate with period $T_{0}$, the remote controller will operate at a slower rate $T=N T_{0}$. The network-induced round-trip time delay between a local controller sending $y_{i}$ and receiving $r_{i}$ will be denoted by $d_{i}(k T)$.

Some assumptions on the values of the delays in each loop are needed. Ample detail will be given in Section V, but at this moment, we may preliminarly assert that, due to the chosen configuration of the Profibus communication, the sync and freeze commands will allow to assume that the delay is coincident in all channels and multiple of the local sampling period. In the sequel, such delay will be denoted by $\delta(k T)=$ $d(k T) \times T_{0}$. The value of $d(k T)$ will be assumed to be known at next sample time, given the sequence of network commands chosen for synchronization (see Sect. V for details).

Although the hierarchical control structure is reminiscent of a cascade control, conventional cascade-control design assumes a time-scale separation so that a separate design is possible [25] and small delays would be negligible in the outer very-low-bandwidth loop. In the proposal here, the action of the remote controller will be fast enough to influence the stability and performance of the inner loop: bandwidth of both loops will be intentionally similar and Lyapunov functions considering the whole remote+local+plant state will be needed.

1) Slow-rate modeling: In order to design the remote controller a slow-rate model (at period $T=N T_{0}$ ) is needed.

Considering the fast-rate behaviour (19), the slow-rate state update equations will be given by the well-known convolution expression [26]:

$$
\begin{gathered}
\chi\left((k+1) N T_{0}\right)=A_{0}^{N} \chi\left(k N T_{0}\right)+ \\
+\sum_{h=1}^{N} A_{0}^{h-1} B_{0} r\left(((k+1) N-h) T_{0}\right)
\end{gathered}
$$

However, as the reference input is updated after a delay of $\delta(k T)=d(k T) T_{0}$ and kept constant until next update (see Figure 6), the above must be corrected to:

$$
\begin{array}{r}
\chi\left((k+1) N T_{0}\right)=A_{0}^{N} \chi\left(k N T_{0}\right)+ \\
+\left(A_{0}^{N-d(k T)} \sum_{j=1}^{d(k T)} A_{0}^{j-1} B_{0}\right) r\left((k-1) N T_{0}\right)+ \\
+\left(\sum_{i=1}^{N-d(k T)} A_{0}^{i-1} B_{0}\right) r\left(k N T_{0}\right)= \\
=A_{0}^{N} \chi\left(k N T_{0}\right)+B_{1}(N, d(k T)) r\left((k-1) N T_{0}\right)+ \\
+B_{2}(N, d(k T)) r\left(k N T_{0}\right)
\end{array}
$$

By assumption, $T$ will be strictly greater than $d(k T) T_{0} \forall k$, because the sampling period $T$ has been selected in order to 


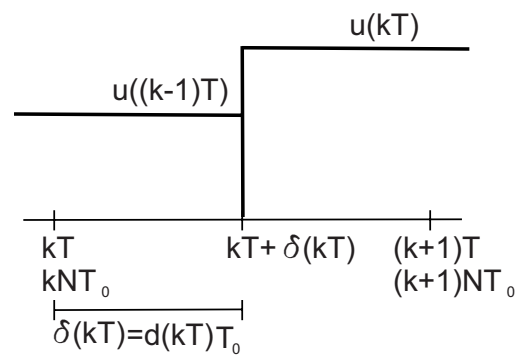

Fig. 6. Control and state update chronogram

verify this restriction ${ }^{4}$. If $d(k T)>N$, the model would get more complex needing incorporation of further past values of $r$, i.e., $r\left((k-2) N T_{0}\right)$, etc. For simplicity, this issue will be pursued no further.

If an augmented state $\Psi(k T)=\left(\chi(k T), r((k-1) T)^{\prime}\right.$ referred to the remote period $T$ is considered, the system as seen by the upper level can be expressed as:

$$
\begin{array}{r}
\Psi((k+1) T)=\left(\begin{array}{cc}
A_{0}^{N} & B_{1}(N, d(k T)) \\
0 & 0
\end{array}\right) \Psi(k T)+ \\
+\left(\begin{array}{c}
B_{2}(N, d(k T)) \\
I
\end{array}\right) r(k T)
\end{array}
$$

For brevity in further developments, notations $A^{*}$ and $B^{*}$ are introduced in (23), yielding:

$$
\begin{array}{r}
\Psi((k+1) T)=A^{*}(N, d(k T)) \Psi(k T)+ \\
+B^{*}(N, d(k T)) r(k T)
\end{array}
$$

The modelling procedure in Section III allows obtaining the above matrices in the experimental platform.

\section{B. Control design strategy}

Once the dual-rate modeling and notation have been described, this section discusses general ideas on the chosen control design methodologies.

Basically, on one hand, three low-order (and hence with low computational requirements) local controllers will be designed using standard mixed-sensitivity $\mathcal{H}_{\infty}$ control techniques [15], assuming a decoupled system.

On the other hand, LMI-gridding and non-stationary Kalman filter are used in the coordinating remote controller (see in [16], [27] the basic formulae for the LMI-gridding approach, and in [28], [29] for the Kalman filter), assuming a fully coupled plant.

In this section, the control techniques in the remote side are reviewed for convenience. Note, importantly, that in order to develop this control system structure, the delay $d(k T)$ must be known. Conditions for applicability of a separation principle are also discussed in [16].

\footnotetext{
${ }^{4}$ For this purpose some experimental off-line tests have been done to determine the maximum round-trip delay.
}

1) LMI gridding: From the augmented model (24), the control synthesis problem can be cast as a state-feedback one, leading to:

$$
r(k T)=-F^{*} \Psi(k T)
$$

however, the gain $F^{*}$ must ensure robustness against the unknown round-trip delay $d(k T)$ because its value is not known a priori at the time $r(k T)$ is computed: its value will be later obtained by using the freeze/sync commands, but it will be useful only for the a posteriori observer part.

In order to achieve stabilizing controllers subject to timevarying delays, an LMI gridding procedure [16] is considered. From [16], if there exist matrices $X$ and $M$ so that (26) on page 7 is verified for any $d(k T)$ which may arise in experimental operation: in this particular application, $d(k T)$ takes values in $[1, \ldots, N-1]$.

If the LMIs are feasible, the feedback controller $F^{*}=$ $M X^{-1}$ stabilizes (24) with decay rate $\vartheta$ (continuous-time equivalent), and $\Psi(k T)^{\mathrm{T}} X^{-1} \Psi(k T)$ is the associated Lyapunov function. As discussed in Section $\mathrm{V}$, the experimental setup required $N=4$ and gridding on possible values of $d(k T)=1,2,3$, so (26) is actually a collection of 3 LMIs.

2) Non-stationary Kalman filter: Regarding the observer design for system (24), as $d(k T)$ can be obtained from freeze/sync commands at the time $r((k+1) T)$ is computed, Kalman gains depending on induced delays can be obtained with non-stationary Kalman filters [30], [31], whose equations are:

$$
\begin{array}{r}
P(k T)=A^{*}(N, d(k T)) \Lambda(k T) A^{*}(N, d(k T))^{T}+V(k T) \\
L(k T)=P(k T) C^{T}\left(C P(k T) C^{T}+W(k T)\right)^{-1} \\
\Lambda((k+1) T)=(I-L(k T) C) P(k T)
\end{array}
$$

Where $V(k T)$ and $W(k T)$ are process and measurement noise parameters. The observer gain is $L(k T)$, with an estimated state, denoted by $\tilde{\Psi}(k T)$, obtained via the currentobserver update equation:

$$
\begin{array}{r}
\tilde{\Psi}(k T)=(I-L(k T) C) \\
\left(A ^ { * } \left(N, d((k-1) T) \tilde{\Psi}((k-1) T)+B^{*}(N, d((k-1) T) r(k T))\right.\right. \\
+L(k T) y(k T)
\end{array}
$$

In order to implement the above equation, the packet received by the remote node should contain the array of measurements $y(k T)$, the time in which those ones were measured, and the delay $d((k-1) T)$ in the preceding cycle as explains next section.

\section{HARDWARE AND NETWORK CONFIGURATION}

The control experiments developed in this paper have been implemented as follows.

In the first level, the local controllers have been implemented using a National Instruments CompactRIO device as shown in Figure 2 (right).

The network was a Profibus-DP one; its nodes were a ComSoft DFProfi-II DP card in the PC and a Profibus module cRio PB in the compactRio controller, the latter operating as a bus master. Details on the network elements can be found in http://www.comsoft.de and http://www.ni.com. 


$$
\left[\begin{array}{cc}
e^{-2 \vartheta T} X & X\left(A^{*}(N, d(k T))\right)^{T}-M^{T}\left(B^{*}(N, d(k T))\right)^{T} \\
A^{*}(N, d(k T)) X-B^{*}(N, d(k T)) M & X
\end{array}\right]>0
$$

TABLE I

DISTRIBUTION HISTOGRAM OF NETWORK ROUND-TRIP DELAY

\begin{tabular}{||l|c|c|c||}
\hline Delay & $5 \mathrm{~ms}$ & $10 \mathrm{~ms}$ & $15 \mathrm{~ms}$ \\
\hline Occurrences & 123,154 & $1,084,502$ & 292,357 \\
\hline Percentage & $8.21 \%$ & $72.3 \%$ & $19.49 \%$ \\
\hline
\end{tabular}

The available Profibus-DP bandwidth enables to use a second level where a remote coordinating control action is injected just every 20 milliseconds. Indeed, the chosen Profibus configuration parameters were bus rate $187.5 \mathrm{kBits} / \mathrm{s}$ with asynchronous operation mode.

Let us now discuss how the remote control and locallevel synchronization are carried out. Every $T=20 \mathrm{~ms}$ the supervisor controller starts the control task as follows (symbollicaly depicted in figure 7):

- Stage 1. Master node sends freeze command to slave nodes. All slaves receive the message at approximately the same time (with some microseconds jitter due to the bus cycle) and freeze the inputs (from the process). In the next Profibus cycle, slaves send frozen inputs (measures) to master node. Receiving freeze command is used also for synchronizing slave clocks to $t=1 \mathrm{~ms}$.

- Stage 2. Master node receives the measures from slaves and process data according to the control algorithm.

- Stage 3. Master node sends sync command to slave nodes. Then, their outputs to the process are internally updated at the next Profibus cycle in the buffers but not applied until an unsync command is received.

- Stage 4. Master node sends the referred unsync command to slaves, and then all of them update the outputs (reference to local controllers) simultaneously at the next multiple of the local sampling time $T_{0}$. As the remote controller knows the moment in which it sent the unsync command, it can compute $d(k T)$ for the observer update.

- Stage 5. With their internal sampling time $T_{0}=5 \mathrm{~ms}$ local controllers are performing platform control algorithm with no communication with master node. At sampling time $T=20 \mathrm{~ms}$ the measurements are taken and stored in the buffer. The master node starts again the control process at $t=21 \mathrm{~ms}$, and the sequence returns to stage 1.

Distribution of round-trip delays $d(k T)$ : An experimental test was conducted to measure the magnitude of the network-induced time delays: the most repeated round-trip time delay corresponds to a $10 \mathrm{~ms}$ period, with eventual delays at 5 and $15 \mathrm{~ms}$, as shown in Table I. For this reason, the grid of times for discretizing the system will be formed by the set of values $(5,10,15) \mathrm{ms}$.

\section{NUMERICAL RESULTS AND EXPERIMENTS}

In this section, the numerical results of the controller computations and the experimental measurements will be described.

Note that, although it is "theoretically possible" to control a linear system with a discrete-time controller at any desired period, the disturbance rejection and tolerance to modeling errors severely diminish as such period increases with unstable systems. This fact is proved to be fundamental in our experiments, in the sense that:

1) the $\mathcal{H}_{\infty}$ controllers designed for the long period $T=$ $20 \mathrm{~ms}$ were not able to stabilize, in actual experiments, even a single maglev, in a local configuration with no network delay, even if they were of course stable in the simulations. Hence, the slow-rate remote-only control option was not a viable solution.

2) On the other hand, a local controller at each of the levitators with a SISO loop may be a viable solution. Indeed, local stabilizing controllers at $T_{0}=5 \mathrm{~ms}$ with the decoupled model were obtained by $\mathcal{H}_{\infty}$ techniques, and were able to stabilise appropriately a single maglev.

3) However, due to disregarded couplings, modeling error is introduced which will degrade performance of the local-only decentralised controllers, as later experiments show. Of course, incorporating the coupling would require communication between the different subsystems, assumed to be feasible only via the chosen network (at a slower rate).

In summary, the above issues justify the need of the proposed dual-rate structure.

In order to deal with these issues, a set of experiments have been developed, as follows.

\section{A. Preliminary experiments: single-maglev control.}

First of all, a preliminary experiment with a single maglev, not with the whole platform, has been developed. In this experiment, first, a standalone $\mathcal{H}_{\infty}$ controller has been designed and implemented. This type of controller is chosen in the local controller in order to suitably balance disturbance rejection performance versus tolerance to modeling errors at particular frequencies.

A first set of controllers are designed for each single maglev using a mixed-sensitivity approach using a set of weight values, that is, sensitivity weight, control action weight and complementary sensitivity weight. For the designed controllers the values of these corresponding weights are 1 , 0.2 and 0.6. Using the Robust toolbox Matlab command $[K, C L, G A M, I N F O]=\operatorname{MIXSYN}(G, 1,0.2,0.6)$, where $G$ is the equivalent discrete model plant for state space maglev model, the different local controllers for each one of the three maglevs are obtained. Weights have been chosen as constants to keep the resulting controller order low.

Experimentally, a set of controllers has been designed using several sampling periods keeping the same design parameters. As a result of all the experiments, the greatest sampling period 


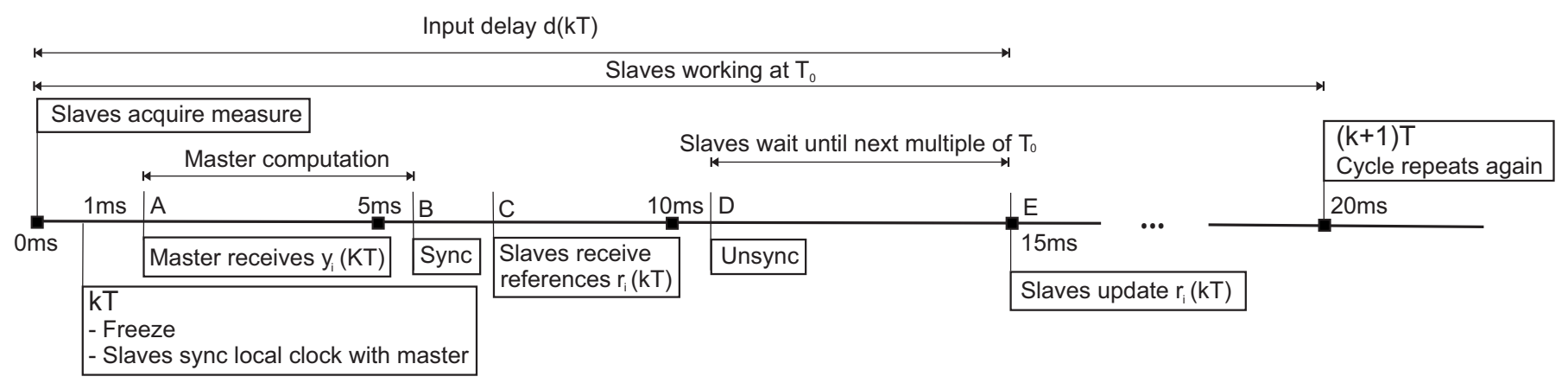

Square mark each $5 \mathrm{~ms}$ indicates time when slave nodes perform periodic control task

Times A, B, C and D are variable anywhere between 5 and $15 \mathrm{~ms}$

Time $\mathrm{E}$ is next multiple of $5 \mathrm{~ms}$ after $\mathrm{D}$ occurs

Fig. 7. Coordination and synchronisation cronogram (PROFIBUS implementation of Figures 5 and 6).

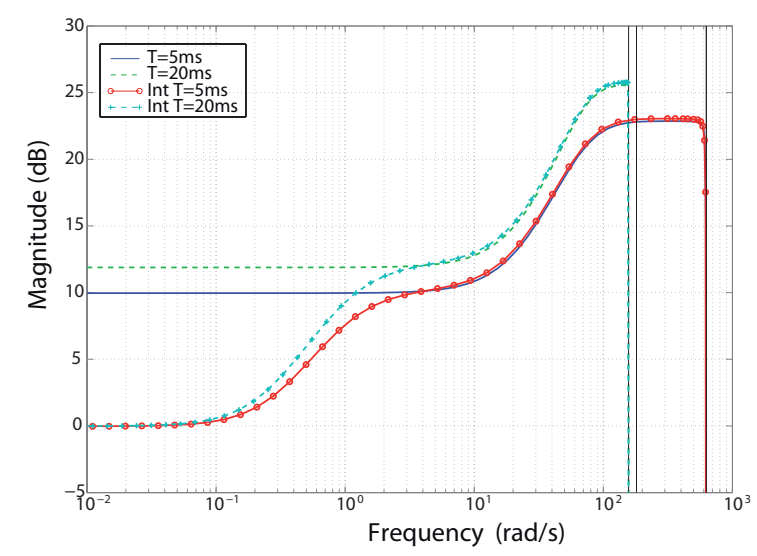

Fig. 8. System's sensitivity to additive uncertainty with different sampling rates.

that can be satisfactorily used with the maglev is $T_{0}=5 \mathrm{~ms}$ : with larger sampling periods, accumulated error can become too large, so it makes the one-maglev system unstable or on the verge of instability.

Fig. 8 shows the additive uncertainty sensitivity analysis, i.e., $K /(1+K G)$, when controller and plant (for a single maglev) are sampled at 5 and $20 \mathrm{~ms}$. As sampling time increases, uncertainty in model and sensitivity increases, making the experimental system unstable.

All the local controllers are very similar, so the numerical values of just one of them is presented below:

$$
G_{R}(z)=\frac{u(z)}{e(z)}=\frac{10.845(z+1)(z-0.878)(z-0.641)}{(z+0.93)\left((z-0.54)^{2}+0.27^{2}\right)}
$$

An additional set of controllers is designed adding an (approximate) integral term. Defining the continuous integrator as $\frac{G(\tau s+1)}{G \tau s+1}$, with large enough $G$ and small enough $\tau$, this continuous term is discretized at $T_{0}$ sampling time using Tustin discretization method. This discretized term is included in the sensitivity weight $\left(W_{I}\right)$ for calculating the new controller. In our case, the resulting weight was $W_{I}(z)=\frac{1.001 z-0.9982}{z-0.9997}$. A new controller is designed using this integrator term in the sensitivity weight (WI) $[K, C L, G A M, I N F O]=\operatorname{MIXSYN}(G, W I, 0.2,0.6)$. As a result, the new set of controllers are obtained. One of them is next presented:

$$
G_{R}(z)=\frac{10.74(z+1)(z-0.99)(z-0.88)(z-0.71)}{(z-1)(z+0.94)\left((z-0.56)^{2}+0.28^{2}\right)}
$$

which, as expected, it is very similar to the above one (31) except at low frequencies.

With these numerical results, a first preliminar singlemaglev experiment is developed, whose output appears in Figure 9. The experiment begins activating the local controller without integrator (see (31)). The system achieves an equilibrium point with position error, as expected, but system performance is suitable. At time $t=10.5 \mathrm{~s}$ controller changes adding the integral term (see (32)) and after a 2 second transient, the system achieves the new equilibrium point with no position error. This experiment is performed with only one standalone maglev. After this experiment is concluded, the platform is assembled with the tree maglevs, and the rest of experiments below are carried-out.

In summary, this preliminary experiment demonstrates that the chosen design parameters for the local controllers allow an adequate SISO control performance for the real plant.

\section{B. Experiment 1: 3-Maglev platform, no-network}

This experiment is developed using the whole platform and the set of local controllers previously developed without integral action (31). Therefore, the network and the supervisor level are not used yet. The experiment starts with platform in equilibrium point, and at time $t=2.5 \mathrm{~s}$ a load change is applied to the platform depositing a 2-euro coin in the center of the triangular aluminum structure. As seen in figure 10, the system response seems to change to a new equilibrium point (i.e., position error) but it barely keeps there and platform becomes unstable and drops at $t=5.5 \mathrm{~s}$.

This is the result of coupling between the different maglevs in the whole platform system, as single-maglev controllers worked perfectly when isolated. 

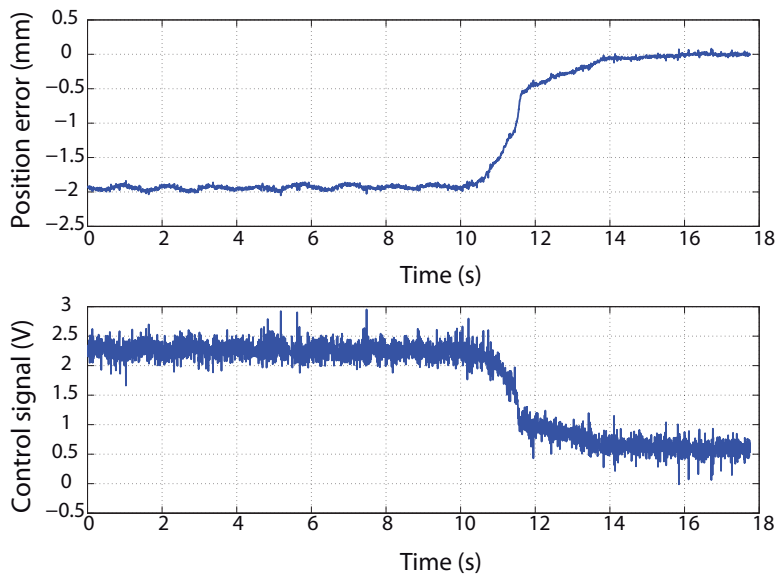

Fig. 9. A single maglev experiment

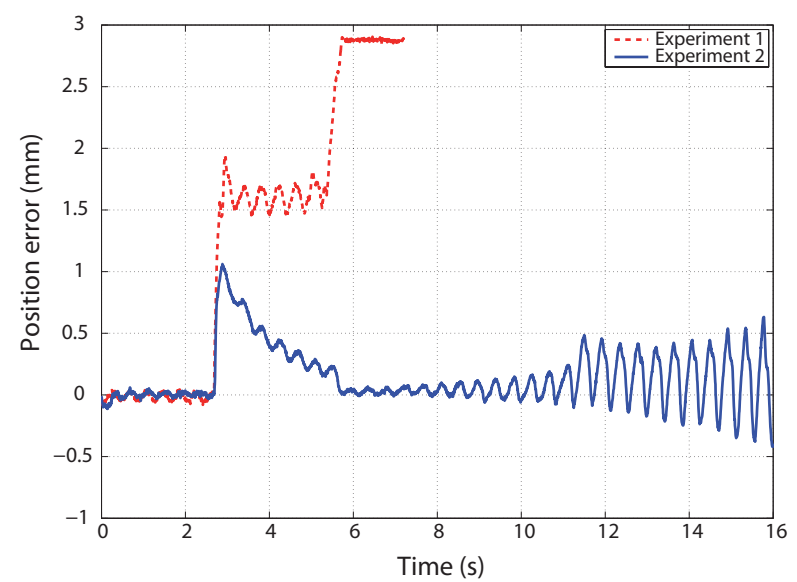

Fig. 10. Platform position in the experiments 1 and 2 .

C. Experiment 2: 3-Maglev platform, no-network, integral action

This experiment is the same as the previous one but using controllers with the integral action included (32). As in the previous experiment, network and supervisor level are not used yet.

Although the integrator tries to recover the reference position, oscillations of increasing amplitude are generated due to the load disturbance and, ultimately, the loop becomes unstable and the platform falls.

With these two experiments (Experiment 1 and Experiment 2 ), coupling in platform is demonstrated to be significative, and it must be taken into account when designing the control system.

\section{Experiment 3: 3-Maglev platform, hierarchical control, no integrator}

This third experiment is developed using the network and an additional supervisor level controller. Each maglev on the platform keeps his own local controller (no integral action) as previously designed, i.e., the same set of controllers used in Experiment 1, see (31).

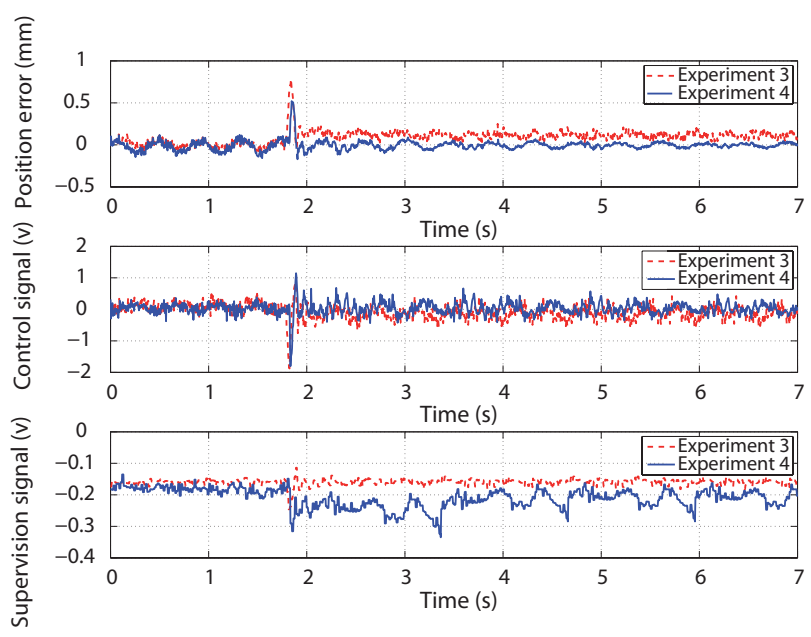

Fig. 11. Figure results for experiments 3 and 4 .

At higher level, in the remote side, a plant model is developed following the results in previous sections, where the considered process is the set composed by the three maglev systems and the corresponding local digital control subsystems with period $T_{0}$.

The resulting state feedback controller (25) obtained has a gain matrix of dimensions $3 \times 21$ as shows (VI-D) depicted on page 10

This supervisor controller is implemented on remote side, jointly with the previously discussed observer.

The experiment starts with platform in equilibrium point, as shown in Figure 11. At time $t=1.75 \mathrm{~s}$ load is applied, and after a transient, system acquires a new, stable equilibrium point, but with position error (as expected). Compared to experiment 1, now the supervisor control level compensates the disturbances introduced by coupling between the three maglevs of the platform.

In figure 11, the top figure shows the position error (center of mass), the middle one shows the control signal applied to maglev, and the bottom one shows the supervision signal generated by the supervisor controller and sent through the network to the local controller. For clarity, only one of the three control and supervision signals are plotted.

\section{E. Experiment 4: 3-Maglev platform, hierarchical control,} integrator (remote)

The results obtained with experiment 3 present position error, so, for obtaining an stable plant without position error, a new remote controller that includes accumulated error in state system is developed. So the controller is designed considering

$$
\begin{aligned}
\left(\begin{array}{c}
\Psi((k+1) T) \\
s((k+1) T)
\end{array}\right)=\left(\begin{array}{cc}
A^{*} & 0 \\
-C^{*} & I
\end{array}\right) & \left(\begin{array}{c}
\Psi(k T) \\
s(k T)
\end{array}\right)+ \\
& +\left(\begin{array}{c}
B^{*} \\
-0
\end{array}\right) r(k T)
\end{aligned}
$$

where the position error in $C^{*} \Psi$ will be zero in steady state [29].

Following this reasoning, the system state vector is expanded adding the accumulated error for each one of the three 


$$
F^{*}=\left(\begin{array}{ccccccccccccccccccccccc}
-5.36 & 383.2 & -0.63 & 44.76 & -0.001 & -0.008 & 0.57 & 0.001 & 0 & 0.22 & 0.12 & -0.013 & \ldots \\
-4.75 & 349.4 & 0.29 & -20.32 & -0.5 & 35.34 & -0.003 & 0.33 & -0.0003 & -0.003 & -0.120 & 0 & \ldots \\
1.963 & 395.7 & -0.11 & -23.14 & -0.19 & 40.08 & 0.0004 & -00002 & 0.06 & 0.0008 & 0.037 & -0.0001 & \ldots \\
& & & & \ldots & 0.001 & 0.044 & 0 & 0.001 & 0.012 & 0 & 0.104 & 0.0004 & 0 & 0 \\
& & & \ldots & 0.194 & -2.45 & -0.008 & -0.001 & -0.02 & 0 & -0.001 & 0.06 & -0.001 \\
& & & \ldots & 0.0001 & 0.007 & 0 & 0.042 & -6.86 & -0.004 & 0.001 & 0 & -0.15
\end{array}\right)
$$

maglevs on the levitated platform. According to this new plant model, the feedback state controller is recalculated obtaining a new state feedback gain, also with LMI gridding.

As the system model vector state has been increased with the new states corresponding to the accumulated errors, the new feedback gain increases their dimensions, being in this case a matrix of size $3 \times 24$.

The new controller with the integral action can keep the platform stable even with load variations. The integral component is working at low rate because it is present in the supervisor level, on the remote side of the controller. As figure 11 shows, after the load is applied to the platform, the system keeps the stable equilibrium point obtaining acceptable results in terms of performance.

\section{CONCLUSIONS}

In this paper a practical Profibus-DP network-based control application has been developed in order to coordinate an unstable triangular maglev platform via a dual-rate controller implementation.

Both local-only and network-only controllers were not able to satisfactorily stabilise the platform. However, a hierarchical control structure succeeded in the experiments: a simple stabilization local control and a refined LMI-based+Kalman Filter solution in the remote side allow to solve the problem caused by limited network bandwidth and time-varying delays.

Even if a particular choice of controller/observer strategy has been experimentally demonstrated, for more demanding applications (out of the scope of this work), the results might be ameliorated by including further theoretical developments such as delay uncertainty (slight desyncrhonization), or delaydependent Lyapunov functions, even incorporating information on the probabilistic distribution of the delays.

\section{REFERENCES}

[1] Y. Tipsuwan and M. Chow, "Control methodologies in networked control systems," Control Eng. Practice, vol. 11, no. 10, pp. 1099-1111, 2003.

[2] Y. Halevi and A. Ray, "Integrated communication and control systems. I- Analysis," ASME, Trans., J. Dynamic Syst., Meas. and Control, vol. 110, pp. 367-373, Dec. 1988.

[3] T. Yang, "Networked control system: a brief survey," IEE Proc. Control Theory Appl., vol. 153, no. 4, pp. 403-412, Jul. 2006.

[4] S. Banerjee, D. Prasad, and J. Pal, "Design, implementation, and testing of a single axis levitation system for the suspension of a platform," ISA transactions, vol. 46, no. 2, pp. 239-246, 2007.

[5] W. Kim, S. Verma, and H. Shakir, "Design and precision construction of novel magnetic-levitation-based multi-axis nanoscale positioning systems," Precision Engineering, vol. 31, no. 4, pp. 337-350, 2007.

[6] W. Kim, K. Ji, and A. Ambike, "Real-time operating environment for networked control systems," vol. 3, no. 3, p. 287, 2006.

[7] J. Paddison, H. Ohsaki, and E. Masada, "Control strategies for maglev electromagnetic suspension bogies," in Decision and Control, 1996., Proceedings of the 35th IEEE, vol. 3, dec 1996, pp. $2796-2797$ vol.3.
[8] R.-J. Wai and J.-D. Lee, "Performance comparisons of model-free control strategies for hybrid magnetic levitation system," Electric Power Applications, IEEE Proceedings -, vol. 152, no. 6, pp. 1556 - 1564, nov. 2005.

[9] P. Holmer, "Faster than a speeding bullet train," IEEE Spectrum, vol. 40, no. 8, pp. 30-34, 2003.

[10] P. Berkelman and M. Dzadovsky, "Large motion range magnet levitation using a planar array of coils," in Proceedings of the 2009 IEEE International Conference on Robotics and Automation. IEEE Press, 2009, pp. 3950-3951.

[11] D. Hristu-Varsakelis and W. S. Levine, Handbook of Networked and Embedded Control Systems. Birkhäuser, 2008.

[12] K. Lee, S. Lee, and M. Lee, "Remote fuzzy logic control of networked control system via Profibus-DP," vol. 50, no. 4, pp. 784-792, 2003.

[13] Z. Lie-ping, Z. Yun-sheng, and Z. Qun-ying, "Remote Control Based on OPC and Profibus-DP Bus," Control Engineering of China, p. 05, 2008.

[14] V. Casanova, J. Salt, A. Cuenca, and V. Mascarós, "Networked control systems over Profibus-DP: simulation model," in 2006 IEEE International Conference on Control Applications, 2006, pp. 1337-1342.

[15] K. Zhou, Essentials of Robust Control. Prentice Hall, 1998.

[16] A. Sala, "Computer control under time-varying sampling period: An LMI gridding approach," Automatica, vol. 41, no. 12, pp. 2077-2082, Dec. 2005.

[17] C. Fernández, M. Vicente, and L. Jiménez, "Virtual Laboratories for Control Education: a Combined Methodology," Int. J. Engng, vol. 21, no. 6, pp. 1059-1067, 2005.

[18] R. Fama, R. Lopes, A. Milhan, R. Galvão, and B. Lastra, "Predictive control of a magnetic levitation system with explicit treatment of operational constraints," in Proceedings of the 18th International Congress of Mechanical Engineering, Ouro Preto, MG,(paper 0560), 2005.

[19] K. Erkan and T. Koseki, "Fuzzy model-based monlinear maglev control for active vibration control systems," International Journal of Applied Electromagnetics and Mechanics, vol. 25, no. 1, pp. 543-548, 2007.

[20] H. Han-hui and T. Qing, "Decoupling fuzzy pid control for magnetic suspended table," Journal of Central South University (Science and Technology), 2009.

[21] N. Al-Muthairi and M. Zribi, "Sliding mode control of a magnetic levitation system," Mathematical Problems in Engineering, vol. 2, no. 2004, pp. 93-107, 2004.

[22] J. E. Marsden and J. Scheurle, "The Reduced Euler-Lagrange Equations," Fields Institute Communications, pp. 139-164, 1992.

[23] J. Van Bladel, Electromagnetic Fields. IEEE WILEY-INTERSCIENCE, 1964.

[24] A. El Hajjaji and M. Ouladsine, "Modeling and nonlinear control of magnetic levitation systems," IEEE Transactions on Industrial Electronics, vol. 48, no. 4, pp. 831-838, 2001.

[25] K. Ogata, Modern Control Engineering. Prentice Hall, 2010.

[26] P. Albertos, "Block multirate input-output model for sampled-data control systems," Automatic Control, IEEE Transactions on, vol. 35, no. 9, pp. 1085-1088, 1990.

[27] A. Sala, "Improving performance under sampling-rate variations via generalized hold functions," Control Systems Technology, IEEE Transactions on, vol. 15, no. 4, pp. $794-797$, july 2007.

[28] R. F. Stengel, Optimal control and estimation. Dover, 1994.

[29] A. P. and A. Sala, Multivariable control systems: an engineering approach. Springer, 2004.

[30] J. Tornero, R. Pizá, P. Albertos, and J. Salt, "Multirate lqg controller applied to self-location and path-tracking in mobile robots," in Intelligent Robots and Systems, 2001. Proceedings. 2001 IEEE/RSJ International Conference on, vol. 2, 2001, pp. 625 -630 vol.2.

[31] M. Mora, R. Pizá, and J. Tornero, "Multirate obstacle tracking and path planning for intelligent vehicles," in Intelligent Vehicles Symposium, 2007 IEEE, june 2007, pp. $172-177$. 
TABLE II

MAGLEV PARAMETERS SUPPLIED BY THE MANUFACTURER.

\begin{tabular}{||c|c|c|c||}
\hline & Maglev 1 & Maglev 2 & Maglev 3 \\
\hline Resistance $\Omega$ & $R_{1}=4.6$ & $R_{2}=5$ & $R_{3}=4.2$ \\
\hline Inductance $\mathbf{~ m H}$ & $L_{1}=52$ & $L_{2}=73$ & $L_{3}=32$ \\
\hline Em. transfer gain N/A & $K_{1}^{1}=-0.56$ & $K_{1}^{2}=-0.7$ & $K_{1}^{3}=-0.82$ \\
\hline Position transfer gain N/m & $K_{2}^{1}=98$ & $K_{2}^{2}=98$ & $K_{2}^{3}=98$ \\
\hline Sensor transfer gain V/m & $K_{3}^{1}=680$ & $K_{3}^{2}=700$ & $K_{3}^{3}=705$ \\
\hline
\end{tabular}

\section{APPENDIX}

\section{A. Magnetic levitation model parameters}

As previously mentioned, some of the maglev parameters have been identified by manufacturer and are listed in the maglev user's manual. Those parameters, for the three maglev's here used, appear in Table II.

As a result of the experimental identification, the values $Q^{1}=Q^{2}=Q^{3}=0.5 \mathrm{Vs} / \mathrm{m}$ have been assigned.

The length of the structure arm $L$ is $0.117 \mathrm{~m}$ (see Fig. 4), and the total weight of platform, including load, $M$ is 0.406 $\mathrm{Kg}$. The inertia matrix:

$$
\left(\begin{array}{lll}
J_{x x} & J_{x y} & J_{x z} \\
J_{y x} & J_{y y} & J_{y z} \\
J_{z x} & J_{z y} & J_{z z}
\end{array}\right)=\left(\begin{array}{ccc}
0.00293 & 0 & 0 \\
0 & 0.00293 & 0 \\
0 & 0 & 0.00541
\end{array}\right)
$$

has been obtained via CAD software (modeling the object with solid geometry, assigning the weights and obtaining inertial data from the CAD analysis module).

Noise matrices used in Kalman filter $V$ and $W$ are diagonal matrices with values 0.0062 and 0.01 respectively. Measure noise $W$ is deduced obtaining sensor measures with the still platform, mechanically fixed. The variations measured about mean value corresponds to noise and this value is used for characterizing the matrix. $V$ characterizes input noises and modeling errors, and it has been adjusted experimentally to obtain a suitable observer dynamics. 